\title{
(6) OPEN ACCESS \\ How does light-intensity physical activity associate with adult cardiometabolic health and mortality? Systematic review with meta-analysis of experimental and observational studies
}

\author{
Sebastien F M Chastin, ${ }^{1,2}$ Marieke De Craemer, ${ }^{2}$ Katrien De Cocker, ${ }^{2,3,4}$ \\ Lauren Powell, ${ }^{5,6}$ Jelle Van Cauwenberg, ${ }^{3,7}$ Philippa Dall, ${ }^{1}$ Mark Hamer, ${ }^{8}$ \\ Emmanuel Stamatakis ${ }^{5,6}$
}

- Additional material is published online only. To view please visit the journal online (http://dx.doi.org/10.1136/ bjsports-2017-097563).

${ }^{1}$ School of Health and Life Science, Institute for Applied Health Research, Glasgow Caledonian University, Glasgow, UK

${ }^{2}$ Department of Movement and Sport Sciences, Ghent University, Ghent, Belgium

${ }^{3}$ Research Foundation Flanders, Brussels, Belgium

${ }^{4}$ Institute for Resilient Regions, University of Southern

Queensland, Toowoomba, Queensland, Australia

${ }^{5}$ Epidemiology Unit, Charles Perkins Centre, University of Sydney, Sydney, New South

Wales, Australia

${ }^{6}$ Prevention Research

Collaboration, School of Public Health, University of Sydney,

Sydney, New South Wales, Australia

${ }^{7}$ Department of Public Health, Ghent University, Ghent, Belgium

${ }^{8}$ School of Sport, Exercise and Health Sciences, Loughborough University, Loughborough, UK

\section{Correspondence to}

Dr Sebastien F M Chastin, School of Health and Life Sciences, Institute for Applied Health Research, Glasgow Caledonian University, Glasgow G4 OBA, UK:

Sebastien.Chastin@gcu.ac.uk

Accepted 26 March 2018

Published Online First

25 April 2018

Check for updates

To cite: Chastin SFM, De Craemer M, De Cocker K et al. Br I Sports Med 2019:53:370-376.

\section{ABSTRACT}

Aim To assess the relationship between time spent in light physical activity and cardiometabolic health and mortality in adults.

Design Systematic review and meta-analysis. Data sources Searches in Medline, Embase, Psyclnfo, CINAHL and three rounds of hand searches.

Eligibility criteria for selecting studies Experimental (including acute mechanistic studies and physical activity intervention programme) and observational studies (excluding case and case-control studies) conducted in adults (aged $\geq 18$ years) published in English before February 2018 and reporting on the relationship between light physical activity ( $<3$ metabolic equivalents) and cardiometabolic health outcomes or all-cause mortality. Study appraisal and synthesis Study quality appraisal with QUALSYST tool and random effects inverse variance meta-analysis.

Results Seventy-two studies were eligible including 27 experimental studies (and 45 observational studies). Mechanistic experimental studies showed that short but frequent bouts of light-intensity activity throughout the day reduced postprandial glucose $(-17.5 \% ; 95 \% \mathrm{Cl}-26.2$ to $-8.7)$ and insulin (-25.1\%; $95 \% \mathrm{Cl}-31.8$ to -18.3$)$ levels compared with continuous sitting, but there was very limited evidence for it affecting other cardiometabolic markers. Three light physical activity programme intervention studies (n ranging from 12 to 58) reduced adiposity, improved blood pressure and lipidaemia; the programmes consisted of activity of $>150 \mathrm{~min} /$ week for at least 12 weeks. Six out of eight prospective observational studies that were entered in the meta-analysis reported that more time spent in daily light activity reduced risk of all-cause mortality (pooled HR $0.71 ; 95 \% \mathrm{Cl} 0.62$ to 0.83 ).

Conclusions Light-intensity physical activity could play a role in improving adult cardiometabolic health and reducing mortality risk. Frequent short bouts of light activity improve glycaemic control. Nevertheless, the modest volume of the prospective epidemiological evidence base and the moderate consistency between observational and laboratory evidence inhibits definitive conclusions.

\section{INTRODUCTION}

Cardiovascular diseases are the leading cause of premature mortality. ${ }^{1}$ Compelling evidence shows a clear dose-response between the total volume of physical activity and risk of cardiovascular diseases and mortality. ${ }^{2-4}$ However, the large majority of the evidence is for leisure-time moderate to vigorous-intensity physical activity (MVPA, defined as $\geq 3$ metabolic equivalents (MET)) improving risk factors associated with cardiovascular diseases such as high blood pressure, overweight and obesity, hyperglycaemia, hyperlipidaemia and mortality. Consequently, health promotion programmes and public health guidelines emphasise the need for the public to engage in MVPA with relatively little consideration to activities of lower intensity. ${ }^{56}$

Light-intensity physical activity (LIPA), activity performed at under 3 METs, including casual walking, doing household shores or activities of daily living, has received much less research attention. However, in the last decade it has been recognised that physical activity represents a continuum. ${ }^{7}$ This raises the question of the health-enhancing potential of LIPA. ${ }^{8}$ LIPA promotion may be a feasible means to increase volume of activity because LIPA does not require dedicated time commitment or planning as it usually involves incidental daily living and increase in movement during occupational time (eg, ambulation, low-intensity walking).

However, there is a paucity of evidence on the effects of LIPA on cardiometabolic health among relatively healthy populations. To date, only one systematic review has attempted to summarise evidence on the relationship between LIPA and cardiometabolic risk factors of cardiovascular disease. ${ }^{9}$ Therefore, we aimed to synthesise evidence from observational and experimental studies and to quantify the effect of LIPA on acute and long-term cardiometabolic health through meta-analysis.

\section{METHODS}

This systematic review is reported following the Preferred Reporting Items for Systematic Reviews and Meta-Analyses ${ }^{10}$ and Meta-analysis of Observational Studies in Epidemiology ${ }^{11}$ guidelines. This review was performed following an a priori written protocol documented in online supplementary material 1.

\section{Literature search}

Four electronic databases (Medline, Embase, PsycInfo and CINAHL) were used to identify relevant studies according to the search strategy which 
was developed in collaboration with two librarians (see online supplementary material 2). The databases were searched from inception to February 2018. As there is no standardised terminology to describe the exposure of interest (light incidental physical activity 1.5-3 METs), we expected difficulties to fully capture the literature comprehensively using a set of keywords. Consequently, a thorough secondary manual search strategy was conducted to ensure completeness of records (see online supplementary material 1 for full search strategy). The reference lists of all selected articles were also screened for eligible records. In turn, the reference lists of any article identified this way were screened as well. This process was repeated until no new eligible articles were identified (three rounds).

\section{Inclusion criteria}

Articles reporting experimental or observational studies investigating the relationship between LIPA and cardiometabolic health markers or all-cause and cardiovascular disease mortality in adults (aged $\geq 18$ years) and published in English were eligible for inclusion. To be included, studies had to measure (either subjectively or objectively) or elicit experimentally LIPA, defined as any physical activity above 1.5 METs and below 3 METs.

\section{Exclusion criteria}

As the focus of the review was on the primary prevention, we excluded studies which investigated cardiac rehabilitation programmes and secondary cardiovascular disease prevention trials and longitudinal studies. For the same reason, we also excluded studies which investigated only populations with diagnosed cardiovascular diseases or patients with cancer.

\section{Study selection}

The title and abstract of retrieved articles were screened for eligibility by two independent reviewers randomly assigned to each article from a pool of five reviewers (SFMC, MDC, JVC, KDC, LP). At this stage, studies were excluded solely if the available information in the title or the abstract made it clear that the article did not meet the criteria. Full texts were retrieved for all remaining articles and were assessed for inclusion by two randomly assigned reviewers to each article from a pool of five reviewers (SFMC, MDC, JVC, KDC, LP) who read the articles independently. Reasons for exclusion were recorded by each reviewer. SFMC collated all information and in the case of disagreement, consensus was reached via discussion between SFMC and the reviewers.

\section{Study quality assessment}

Included studies were rated independently by pairs of reviewers from a pool of six coauthors (LP, JVC, MDC, PD, KDC) using the Standard Quality Assessment Criteria for Evaluating Primary Research Papers from a Variety of Fields QUALSYST tool. ${ }^{12}$ This tool was selected as it allows appraising quality and assessing potential for bias over a wide variety of research designs from experimental to observational. SFMC collated all information and in the case of disagreement, consensus was reached via discussion.

\section{Data extraction}

Study characteristics and data describing the relationship between LIPA and cardiometabolic outcomes and mortality were extracted using different pro forma for experimental and observational studies. Each study was assigned randomly a pair of reviewers from a pool of five coauthors (SFMC, LP, JVC, MDC, KDC) to extract the information.

\section{Meta-analysis}

We made an a priori decision to perform a meta-analysis only if at least four studies were eligible for a given outcome and the research design would allow effect sizes to be harmonised. If two articles reported results drawn from the same experimental study or observational data set we only included the study with the highest quality score in the meta-analysis. In order to ascertain if there was a differential benefit of LIPA for people who have some form of metabolic impairment (eg, obesity or type 2 diabetes) we conducted subgroup analysis by metabolic health status of participants (studies that excluded participants with metabolic impairment; studies that specifically recruited participants free of such impairments). Meta-analyses were performed with Review Manager V.5.3 $3^{13}$ using the inverse-variance methods with a random effect because of the small number of studies available and their methodological diversity. Heterogeneity was measured using Cochran's $\mathrm{X}^{2}$ test and Higgins's $\mathrm{I}^{2}$ test. Publication bias was explored using funnel plots. We conducted sensitivity analysis by removing the lowest quality studies from the analysis.

\section{RESULTS}

\section{Study selection and characteristics}

The systematic search in the databases and the hand search yielded 6683 articles. Of these, we screened 321 full texts and finally included 72 in the review (figure 1). The majority of articles were excluded due to the way LIPA was defined, for example, LIPA often being defined as a low volume of activity regardless of intensity. The characteristics of the included studies are detailed in online supplementary tables S1-S4. Publication dates ranged from 1990 to 2018, with 50\% of included studies published in the last 4 years. In the included articles, 27 reported on experimental studies, including 20 acute mechanistic studies and seven physical activity programme intervention studies. The remaining 45 articles reported on observational studies, including 28 cross-sectional and 17 prospective analyses. Cardiometabolic and other health outcomes considered in the studies included adiposity markers (body mass index, waist circumference), glycaemia (plasma glucose, HbA1c, homeostasis model assessment for insulin, plasma insulin, C-peptides), lipidaemia (plasma triglycerides, high-density lipoprotein and low-density lipoprotein cholesterol), blood pressure, inflammatory markers (eg, C-reactive protein), fitness, incidence of chronic diseases (diabetes, cardiovascular disease, cancers) and all-cause mortality. The majority of mechanistic acute studies concentrated on glycaemia and lipidaemia while physical activity intervention programme studies most often examined blood pressure. Cross-sectional studies primarily investigated glycaemia, lipidaemia and adiposity, while prospective studies commonly focused on mortality.

\section{Study quality}

Quality scores for each study are given in online supplementary tables S1-S4. Overall quality scores were normally distributed with a mean of $65 \%$ in each type of study. The majority of controlled studies used quasiexperimental or crossover designs rather than randomised controlled trials. Among the acute mechanistic studies, articles reporting experiments conducted in free-living conditions scored lower than those conducted in controlled laboratory settings. For physical activity programme 


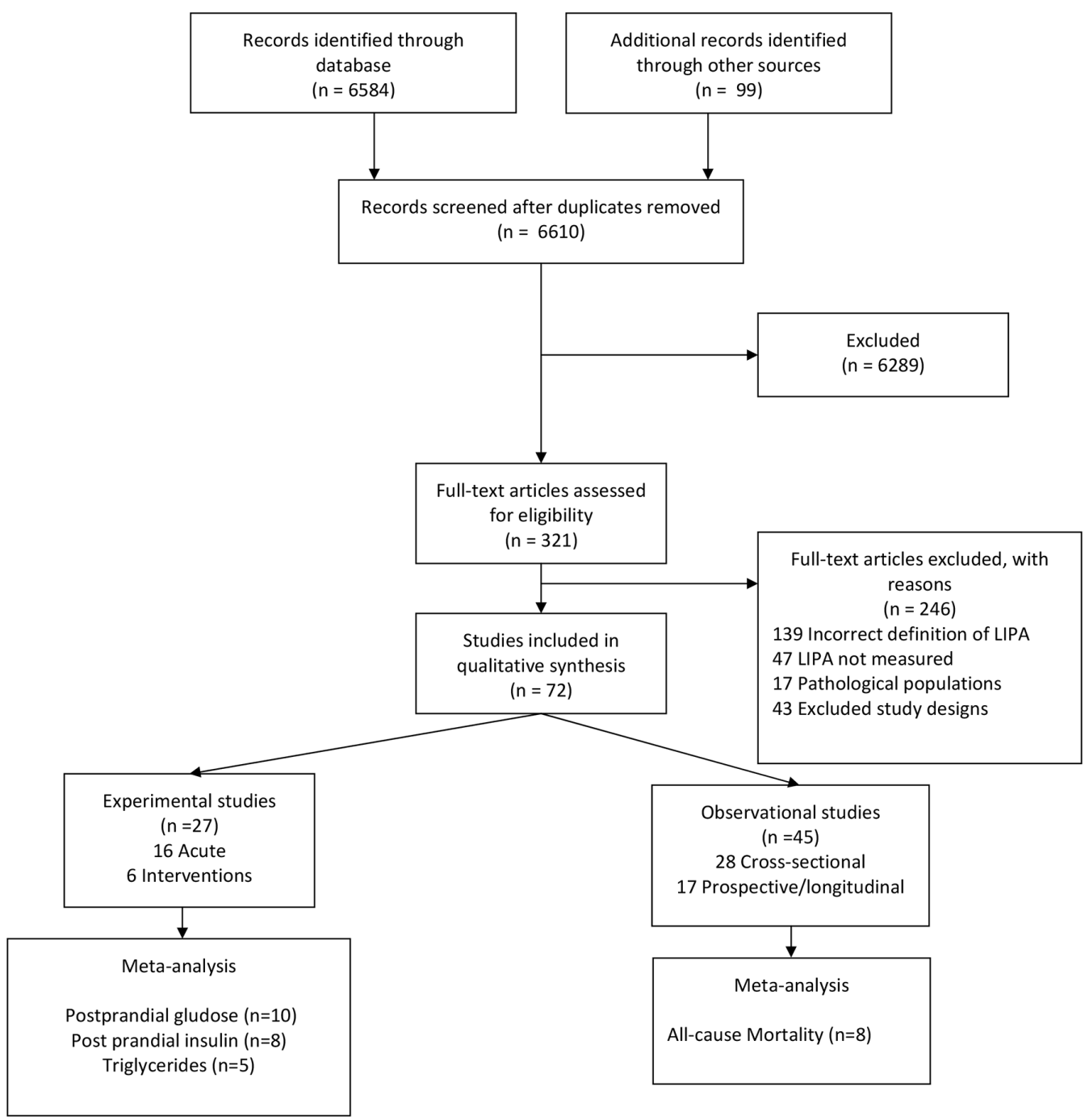

Figure 1 Preferred Reporting Items for Systematic Reviews and Meta-Analyses (PRISMA) diagram. LIPA, light-intensity physical activity.

intervention studies, small sample sizes and lack of an appropriate control group were the most common reasons for lower quality scores. The range of scores was wider for observational studies mostly due to small sample sizes and inadequately justified analytic approaches.

\section{Acute mechanistic studies}

Twenty crossover studies investigated the acute effect through the course of a day of exposure to bouts of LIPA. Four studies investigated the effect of a single continuous bout of LIPA, ${ }^{14-17}$ while 12 studies examined the effect of a set sequence of short bouts in laboratory settings, ${ }^{18-29}$ and 4 studies examined the effect of the natural sequence of bouts of LIPA in free living conditions. ${ }^{30-33}$ Among all mechanistic studies, 13 investigated the effect on glycaemia, ${ }^{172022-26283032-34} 6$ on triglycerides, ${ }^{19} 20243233355$ on cholesterol, ${ }^{1723303233} 5$ on blood pressure 2123293336 and 1 specifically on inflammatory markers. ${ }^{15}$ Sample sizes ranged from $10^{26}$ to $34^{28}$ participants, either healthy or drawn from convenience samples of metabolically impaired populations.

Meta-analysis was possible for studies investigating the effect of frequent short bouts of LIPA on plasma glucose, insulin and triglycerides (figure 2). Overall, acute mechanistic studies resulted in a statistically significant reduction in postprandial glucose $(-17.48 \%$; $95 \% \mathrm{CI}-31.74$ to -8.42$)$ induced by bouts of LIPA as short as $2 \mathrm{~min}$ and interrupting sedentary behaviour at least every $20 \mathrm{~min}$ (figure 2A). Subgroup analysis showed that the effects on glucose outcomes were only significant both in metabolically impaired individuals. However, heterogeneity was significant $(\mathrm{p}<0.001)$ and high $\left(\mathrm{I}^{2}=95 \%\right)$. This heterogeneity was driven by the inclusion of studies conducted both in the laboratory and in free-living conditions. ${ }^{3032} 33$ If these studies were excluded, the four studies on metabolically impaired participants were homogeneous $\left(\mathrm{I}^{2}=0 \%\right)$ and statistically insignificant $(p=0.54)$. Sensitivity analysis showed that when the lowest quality study conducted in free-living conditions was removed from the analysis, results for healthy individual became significant $(p=0.0005)$. There was no statistical difference in effect between groups but the effect appears larger in metabolically impaired individuals. Among laboratory studies, short bouts of LIPA reduced postprandial glucose by $20.07 \%$ (95\% CI -37.18 to -2.96$)$ for normal individual and by $-28.72 \%$ (95\% CI -33.92 to -23.52$)$ in impaired individuals.

For insulin (figure 2B), experimental studies displayed a statistically significant difference in postprandial insulin level $(-25.05 ; 95 \%$ CI -31.76 to -18.33$)$ with no difference in effect between groups. These results persisted in subsequent sensitivity analyses where lower quality or free-living studies were removed from the analysis. In addition, two studies ${ }^{16} 37$ 


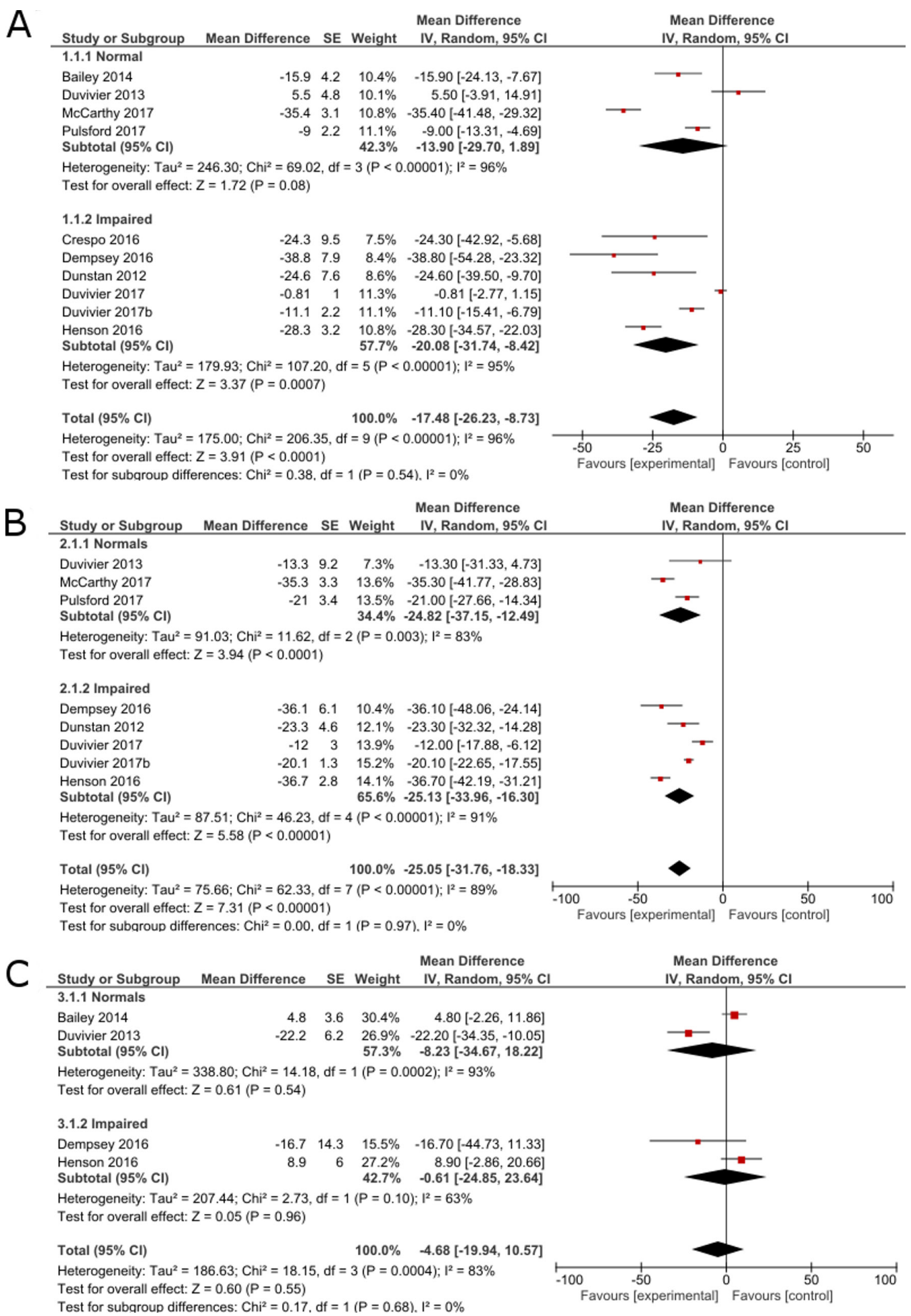

Figure 2 Forest plot for inverse-variance meta-analysis of acute mechanistic studies showing the effect of frequent short bouts of light activity on (A) postprandial glucose level, (B) postprandial insulin level and (C) triglyceride levels, expressed as percentage change compared with the control condition consisting of a whole day (>7 hours) sitting without interruptions.

also reported that the beneficial effect of LIPA on glycaemia persisted until the following day. Heterogeneity was high and significant $\left(\mathrm{p}<0.001 ; \mathrm{I}^{2}=89 \%\right)$ and was not modified by the sensitivity analysis.

For triglycerides (figure 2C), there was no significant effect $(-0.31 \% ; 95 \%$ CI -5.62 to 5.01$)$. Subgroup analysis showed no difference between healthy and metabolically impaired individuals. Heterogeneity was high and statistically significant $\left(\mathrm{p}<0.001 ; \mathrm{I}^{2}=83 \%\right)$. The sensitivity analysis did not alter any of the results but did reduce the heterogeneity, $\mathrm{I}^{2}=27 \%$.

Finally, three out of four studies investigating blood pressure reported significant positive effects of LIPA ${ }^{19} 2131$ while one reported no significant effects. ${ }^{38}$ There were no significant effects of LIPA on inflammatory markers. ${ }^{15}$ 
Physical activity programme intervention studies

Seven studies investigated the effect of participating in a longterm physical activity programme of LIPA on fitness $(n=1)$, lipidaemia $(n=4)$, adiposity $(n=4)$, glycaemia $(n=5)$ and blood pressure $(n=4)$. There were not enough studies with outcomes data that could be pooled to conduct a meta-analysis and only a narrative synthesis was possible. LIPA was delivered in the form of walking programmes, cycling or yoga for 12-16 weeks at a volume ranging from 90 to $300 \mathrm{~min} /$ week. Three studies were quasiexperimental ${ }^{39-41}$ with small sample sizes ranging from 8 to 15 participants, and four were randomised controlled trials ${ }^{42-44}$ with larger sample sizes ranging from 25 to 171 participants. Quasiexperimental studies reported significant improvements in fitness (increase of $5.5 \%$ in $\mathrm{VO}_{2 \max }{ }^{39}$ ), blood pressure (reduction of around $10 \%$ in systolic and diastolic blood pressure), ${ }^{41}$ glycaemia (33\% increase in glucose effectiveness ${ }^{39} 40$ ), adiposity (reduction of $6.1 \%$ in body $\mathrm{fat}^{41}$ ) and lipidaemia (reduction of $6 \%$ in total cholesterol $^{41}$ ) as a result of engaging in LIPA structured programmes. However, these results were not systematically replicated in larger scale randomised trials. Only Paoli et $\mathrm{al}^{42}$ reported a significant improvement in adiposity (reduction of $9.7 \%$ in fat mass), blood pressure (decrease of $8 \%$ in systolic blood pressure and 2.5 in diastolic blood pressure) and lipidaemia (decrease of $2.5 \%$ in total cholesterol) after 12 weeks of $150 \mathrm{~min} /$ week of LIPA delivered as a light-intensity circuit training programme. The other three studies ${ }^{43-45}$ showed no effect, but the volume of LIPA delivered was considerably lower at $90 \mathrm{~min} /$ week of LIPA.

\section{Cross-sectional observational studies}

Twenty-eight articles included in this review reported results of cross-sectional analyses, including nine articles of the 20032006 waves of the National Health and Nutrition Examination Survey (NHANES) and another 19 other epidemiological studies (online supplementary table S3). There was substantial heterogeneity in outcome measures and analytic procedures to enable meta-analysis. Among those, 17 studies reported associations with adiposity markers, 14 with lipidaemia markers, 19 with glycaemia markers, 3 with cardiophysical fitness, 7 with inflammatory markers, 9 with blood pressure and 5 with metabolic syndrome. Sample sizes ranged from $n=50$ to 11000 . Most articles $(n=17)$ were based on objectively measured LIPA. There were consistent reports across studies of an association between time spent in LIPA and adiposity markers, lipidaemia markers (particularly triglyceride levels) and inflammatory markers. Generally, the reported effect sizes were small and were consistently stronger with the increased absolute intensity of LIPA considered. Evidence concerning associations with glycaemia was equivocal. Five separate analyses of NHANES data systematically reported beneficial associations between time spent in LIPA and glucose or insulin levels. This strongly suggests that in this study this cross-sectional association is indeed present, but this was not replicated in all other studies which mostly reported non-significant for association between time spent in LIPA and glycaemia. Equivocal findings were also found for outcomes such as fitness, the metabolic syndrome and composite metabolic scores. For blood pressure, the evidence was particularly inconsistent, even between separate analyses of NHANES data.

\section{Prospective studies}

Eligible articles included 17 prospective studies (online supplementary table 54$)$. The majority of those $(n=12)$ reported associations between LIPA and all-cause mortality, including four different analyses of the NHANES data set. ${ }^{46-50}$ Five articles studied the association between LIPA and incidence of cardiometabolic diseases (type 2 diabetes and metabolic syndrome), one investigated change in glycaemia and one studied the change in adiposity. No relationship was found between time spent in LIPA and metabolic syndrome. ${ }^{51}$ Mekary et al showed a small decrease in body weight to be associated with increase in time spent in LIPA. ${ }^{52}$ A significant reduction in risk of developing type 2 diabetes (HR $0.60 ; 95 \%$ CI 0.40 to 0.91 ) was reported with higher time spent in LIPA in a study of over 14000 Finnish men and women, ${ }^{53}$ but no significant longitudinal improvements in glycaemia and insulin resistance were found in a much smaller study of $n=192$ participants. ${ }^{54}$ In contrast with these mixed results, studies with all-cause mortality as the outcome overwhelmingly reported significant beneficial associations. Results from five mortality studies could be pooled into a meta-analysis. The results presented in figure 3 show a pooled 29\% reduction (95\% CI $-17 \%$ to $-38 \%$ ) in HR of mortality for longer time spent in LIPA (equivalent to a $29 \%$ reduction in HR for doubling the time spent in LIPA). Sensitivity analysis of the meta-analysis revealed that this result was robust. The results heterogeneity was moderate but significant $\left(\mathrm{p}=0.05 ; \mathrm{I}^{2}=57 \%\right)$. The heterogeneity can be traced to the two studies which used self-reported LIPA time ${ }^{5556}$ rather than objective measures in the other three. It should be noted that three potentially eligible studies ${ }^{46-48}$ were not included in the meta-analysis. Three of them were excluded $^{46-48}$ because they used the same NHANES data set as Matthews et al's study ${ }^{50}$ which scored higher in terms of

Figure 3 Forest plot for inverse variance meta-analysis of prospective studies showing the association between time spent in light activity and allcause mortality expressed as HR between highest and lowest amount of time reported in the studies. This corresponds to about a doubling of the time spent in light activity daily. 
quality rating. These three studies showed different results ${ }^{46-48}$ for the same data set suggesting that conclusions are sensitive to different analytical approaches.

\section{Risk of bias and heterogeneity}

No major risk of bias was detected using QUALSYST or on examination of the funnel plots (online supplementary figures S1-S4). These show some asymmetry but it is unlikely to be due to publication bias. This heterogeneity is more probably due to methodological and sample heterogeneity in the studies considered than a real heterogeneity in results. Sources of methodological heterogeneity are the mixed methods used to measure light activity as studies included used both subjective and objective measures. In addition, different cut-point and thresholds were used to define the upper limit of light activity as 3 METs, between studies.

\section{DISCUSSION}

This is the first systematic review and meta-analysis to investigate the relationship between LIPA and cardiometabolic health and all-cause mortality across a range of research designs. While relatively limited in volume, the evidence examining the relationship between LIPA and cardiometabolic outcomes points towards acute positive effects on glucose and insulin and possibly all-cause mortality risk reduction. There seems to be limited evidence suggesting such effects on lipids and other cardiovascular risk factors. The observational evidence is less clear with the large majority of studies being cross-sectional while the limited number of prospective studies points towards beneficial associations with cardiovascular risks.

In spite of these limitations of the evidence base the results of our overall synthesis offer some support to the notion that submoderate intensities of physical activity have mortality and perhaps cardiovascular risk reduction properties. Few studies directly contrasted the effect size of LIPA and MVPA but overall the effects reported for LIPA are between two and four times smaller than for the same amount of time spent in MVPA. For example, Matthews et al ${ }^{49}$ found an HR for mortality of 0.68 for an increase of 1 hour of LIPA compared with the reference group while it was 0.33 for 1 hour of MVPA. Similarly, experimentally Paoli et $a l^{42}$ found between two and four times stronger effects for MVPA in their physical activity intervention programme.

MVPA appears to have a more potent effect on cardiometabolic health than LIPA per unit of time. However, various barriers to achieving the minimum recommended $150 \mathrm{~min}$ of MVPA per week may make LIPA an easier to initiate option for certain population groups, including elderly, frail, some functionally compromised individuals, and those who are particularly inactive. It seems that the effect of light activity might be stronger in metabolically impaired individuals, although the difference was not statistically significant. In addition, the study by McCarthy et $\mathrm{al}^{28}$ reports that fitness moderates the effect of light activity, suggesting again that light activity might be more beneficial to impaired individuals. The theoretically, at least, potential of LIPA is that it might be more feasible and accessible, requiring less motivation for largest part of the population. Most LIPA is incidental to daily living and does not require high level of starting fitness or exercise skills. While it is less potent than MVPA there is potentially a larger 'time reservoir' to tap into. LIPA might be a pragmatic additional target for future interventions and public health guidelines.

Interestingly, the move towards promoting moderate-intensity activity through the public health guidelines ${ }^{57}$ was initially also a pragmatic decision with feasibility and accessibility at its core. At the time, while the evidence from exercise physiology showed that vigorous activity had optimal physiological effects, it was felt that the promotion of moderate-intensity activities like brisk walking was more likely to be feasible for larger parts of the population. Ever since, guidelines and interventions have been encouraging individuals to make up the deficit in activity volume during their leisure time through engaging in MVPA. From the public health perspective and assuming that the LIPA evidence base will develop adequately in the years to come, a similar feasibility-centred argument could be extended to LIPA.

\section{Biological mechanisms}

The results of our review should be viewed through the limited mechanistic information explaining any observed associations and effects of LIPA in humans. A previously proposed animal-based mechanism stated that, compared with muscular inertia, there is substantial increase in lipoprotein lipase activity during light ambulation, suggesting that LIPA might be facilitating better metabolism of circulating lipids. ${ }^{58}$ This mechanism was proposed in $2003^{58}$ but has not been verified in humans and as such it is unknown if it can explain the epidemiological and experimental literature findings on the whole. Sedentary behaviour-focused paradigms that could lend mechanistic insights of the vascular, cellular and metabolic LIPA pathways of action are also still at hypothesis level. ${ }^{58}$

\section{Implications for practice}

Increase in LIPA can occur through activities of daily living at work, home and during transportation and could potentially be achieved with less onus on the individual by promoting a physical environment and culture conducive to movement and activity. Our review suggests that maintaining or/and increasing daily levels of LIPA may have health-promoting potential. With very few exceptions, ${ }^{59}$ current guidelines ${ }^{56}$ do not explicitly recommend LIPA because at the time of their development there was a near absence of evidence. Our review provides the first synthesis consolidating evidence from different studies on the potential of the largest time component of daily physical activity for primary prevention. However, the evidence remains currently limited.

\section{Implications for research}

Our results showed that LIPA may have beneficial health effects but also highlighted important gaps of the evidence base. Ascertaining the dose-response relationships between volume of LIPA and different health outcomes will require careful long-term experimentation to provide targets for intervention and policy. Greater attention needs to be directed to interactions with time spent in other components of the 24-hour physical activity continuum, such as MVPA and sleep sedentary behaviours. This may require novel analytical approaches capable of dealing with the compositional nature of the whole 24 hours. ${ }^{60}$ The body of evidence of acute experimental trials showing consistent effects of LIPA bouts on insulin and glucose (figure 2) provides useful direction for future mechanistic studies. Advances in objective physical activity measures and more accurate activity type classification and recognition are likely to improve substantially our understanding on health attributes of LIPA in the near future.

\section{Study strengths and limitations}

The main strength of this study is that it considers a wide range of evidence from acute effects to long-term exposure. The main limitation of this review and meta-analysis is the relatively small amount of evidence found for some designs and its heterogeneity. 
Indeed, heterogeneity metrics for the meta-analyses are all high, which calls for caution in drawing quantitative conclusions. However, this is not surprising considering the relative novelty of this line of investigation. One of the main difficulties is the lack of a standardised definition and operationalisation of LIPA, which might be addressed in the future with more standardised definitions. ${ }^{60}$ The comprehensive search methodology that includes multiple rounds of manual searches deployed to address this issue is another strength of this study.

\section{CONCLUSION}

Our combined meta-analytical and best evidence synthesis found some evidence that light-intensity activity is associated with beneficial acute and long-term cardiometabolic responses and lower risk of mortality. Nevertheless, the moderate consistency between observational and laboratory evidence and the absence of an established biological mechanism inhibits definitive conclusions. Future research should seek to further build an evidence base that will confidently inform public health and clinical recommendations on submoderate physical activity intensities. Essential steps towards this goal will be to (A) shed further light on the long-term health effects and mechanisms of light-intensity activity and (B) identify optimal ways to promote light-intensity activity for public health purposes.

What is already known?

- There is an L-shaped dose-response between total volume of physical activity and health.

- Leisure-time moderate to vigorous-intensity physical activity is recommended by most public health guidelines.

\section{What are the new findings?}

- Frequent short bouts of light-intensity activity may have acute beneficial physiological effects, particularly in individuals with metabolic impairments.

- There is a moderate amount of consistent (mostly crosssectional) epidemiological evidence showing that more time spent daily in light-intensity activity is associated with better cardiometabolic health outcomes; and some prospective epidemiological evidence that points towards decreased risks of all-cause mortality.

\section{Implication of practice}

- In addition to meeting established guidelines for moderate to vigorous physical activity, practitioners could consider advising patients to increase daily light physical activity.

- In situations where moderate-intensity activity is not a feasible option, there is merit to shifting the onus from encouraging individuals to partly make up the deficit of physical activity during their leisure time with more movement in daily lives.

Correction notice This article has been corrected since it was published Online First. The reference list has been updated, and other minor corrections in the text have been implemented.

Acknowledgements The authors acknowledge Juan Pablo Rey-López for his input and help developing this manuscript. KDC is supported by a postdoctoral fellowship of the Research Foundation Flanders (FWO11/PDO/097). JVC is supported by a postdoctoral fellowship of the Research Foundation Flanders (FWO 1211117N). $\mathrm{MH}$ acknowledges support from the National Institute for Health Research (NIHR)
Leicester Biomedical Research Centre, which is a partnership between University Hospitals of Leicester NHS Trust, Loughborough University and the University of Leicester. ES is supported by the National Health and Medical Research Council through a Senior Research Fellowship, and by a University of Sydney SOAR Fellowship.

Contributors SFMC led the review and meta-analysis and wrote the manuscript. $\mathrm{ES}$ and $\mathrm{MH}$ contributed to the design, hand searches and revisions of the manuscript MDC, KDC, JVC, PD and LP contributed to the selection, appraisal, data extraction of the article and revisions of the manuscript. LP conducted the initial searches and updated the searches.

Funding The authors have not declared a specific grant for this research from any funding agency in the public, commercial or not-for-profit sectors.

Competing interests None declared.

Patient consent Not required.

Provenance and peer review Commissioned; externally peer reviewed.

Open access This is an open access article distributed in accordance with the Creative Commons Attribution Non Commercial (CC BY-NC 4.0) license, which permits others to distribute, remix, adapt, build upon this work non-commercially, and license their derivative works on different terms, provided the original work is properly cited and the use is non-commercial. See: http://creativecommons.org/ licenses/by-nc/4.0/

(c) Article author(s) (or their employer(s) unless otherwise stated in the text of the article) 2019. All rights reserved. No commercial use is permitted unless otherwise expressly granted.

\section{REFERENCES}

1 World Health Organisation. Global status report on noncommunicable diseases 2014 176. Geneva: World Health Organization, 2014

2 Kyu HH, Bachman VF, Alexander LT, et al. Physical activity and risk of breast cancer, colon cancer, diabetes, ischemic heart disease, and ischemic stroke events: systematic review and dose-response meta-analysis for the global burden of disease study 2013. BMJ 2016:354:i3857.

3 Samitz G, Egger M, Zwahlen M. Domains of physical activity and all-cause mortality: systematic review and dose-response meta-analysis of cohort studies. Int J Epidemiol 2011;40:1382-400.

4 Arem H, Moore SC, Patel A, et al. Leisure time physical activity and mortality: a detailed pooled analysis of the dose-response relationship. JAMA Intern Med 2015;175:959-67.

5 Haskell WL, Lee IM, Pate RR, et al. Physical activity and public health: updated recommendation for adults from the American College of Sports Medicine and the American Heart Association. Med Sci Sports Exerc 2007;39:1423-34.

6 Department of Health. Start active, stay active: a report on physical activity from the four home countries' Chief Medical Officers. 2011.

7 Wannamethee SG, Shaper AG. Physical activity in the prevention of cardiovascular disease: an epidemiological perspective. Sports Med 2001;31:101-14.

8 Levine JA, Schleusner SJ, Jensen MD. Energy expenditure of nonexercise activity. Am J Clin Nutr 2000;72:1451-4.

9 Batacan RB, Duncan MJ, Dalbo VJ, et al. Effects of light intensity activity on cvd risk factors: a systematic review of intervention studies. Biomed Res Int 2015;2015:1-10.

10 Hutton B, Salanti G, Caldwell DM, et al. The PRISMA extension statement for reporting of systematic reviews incorporating network meta-analyses of health care interventions: checklist and explanations. Ann Intern Med 2015;162:777-84

11 Stroup DF, Berlin JA, Morton SC, et al. Meta-analysis of observational studies in epidemiology: a proposal for reporting. Meta-analysis Of Observational Studies in Epidemiology (MOOSE) group. JAMA 2000;283:2008-12.

12 Kmet LM, Lee RC, Cook LS. Standard quality assessment criteria for evaluating primary research papers from a variety of fields. Edmonton: Alberta Heritage Foundation for Medical Research (AHFMR). HTA Initiat 2004;13.

13 Higgins JPT, Green S. Cochrane handbook for systematic reviews of interventions. Australia: Cochrane Training, 2011.

14 Schwarz AJ, Brasel JA, Hintz RL, et al. Acute effect of brief low- and high-intensity exercise on circulating insulin-like growth factor (IGF) I, II, and IGF-binding protein-3 and its proteolysis in young healthy men. J Clin Endocrinol Metab 1996;81:3492-7.

15 Mendham AE, Donges $C E$, Liberts $E A$, et al. Effects of mode and intensity on the acute exercise-induced IL-6 and CRP responses in a sedentary, overweight population. Eur J Appl Physiol 2011;111:1035-45.

16 Mestek ML, Plaisance EP, Ratcliff LA, et al. Aerobic exercise and postprandial lipemia in men with the metabolic syndrome. Med Sci Sports Exerc 2008;40:2105-11.

17 Newsom SA, Everett AC, Hinko A, et al. A single session of low-intensity exercise is sufficient to enhance insulin sensitivity into the next day in obese adults. Diabetes Care 2013;36:2516-22.

18 Thorp AA, Healy GN, Winkler E, et al. Prolonged sedentary time and physical activity in workplace and non-work contexts: a cross-sectional study of office, customer service and call centre employees. Int J Behav Nutr Phys Act 2012;9. 
19 Dempsey PC, Sacre JW, Larsen RN, et al. Interrupting prolonged sitting with brief bouts of light walking or simple resistance activities reduces resting blood pressure and plasma noradrenaline in type 2 diabetes. J Hypertens 2016;34:2376-82.

20 Dempsey PC, Larsen RN, Sethi P, et al. Benefits for type 2 diabetes of interrupting prolonged sitting with brief bouts of light walking or simple resistance activities. Diabetes Care 2016:39:964-72.

21 Larsen RN, Kingwell BA, Sethi P, et al. Breaking up prolonged sitting reduces resting blood pressure in overweight/obese adults. Nutr Metab Cardiovasc Dis 2014;24:976-82.

22 Pulsford RM, Blackwell J, Hillsdon M, et al. Intermittent walking, but not standing, improves postprandial insulin and glucose relative to sustained sitting: A randomised cross-over study in inactive middle-aged men. J Sci Med Sport 2016;3.

23 Bailey DP, Locke CD. Breaking up prolonged sitting with light-intensity walking improves postprandial glycemia, but breaking up sitting with standing does not. J Sci Med Sport 2015;18:294-8.

24 Henson J, Davies MJ, Bodicoat DH, et al. Breaking up prolonged sitting with standing or walking attenuates the postprandial metabolic response in postmenopausal women: a randomized acute study. Diabetes Care 2016;39:130-8.

25 Dunstan DW, Kingwell BA, Larsen $\mathrm{R}$, et al. Breaking up prolonged sitting reduces postprandial glucose and insulin responses. Diabetes Care 2012;35:976-83.

26 Crespo NC, Mullane SL, Zeigler ZS, et al. Effects of standing and light-intensity walking and cycling on 24-h glucose. Med Sci Sports Exerc 2016;48:2503-11.

27 Grace MS, Dempsey PC, Sethi P, et al. Breaking up prolonged sitting alters the postprandial plasma lipidomic profile of adults with type 2 diabetes. J Clin Endocrinol Metab 2017;102:1991-9.

28 McCarthy M, Edwardson CL, Davies MJ, et al. Fitness moderates glycemic responses to sitting and light activity breaks. Med Sci Sports Exerc 2017;49:2216-22.

29 Zeigler ZS, Mullane SL, Crespo NC, et al. Effects of standing and light-intensity activity on ambulatory blood pressure. Med Sci Sports Exerc 2016;48:175-81.

30 Duvivier BM, Schaper NC, Bremers MA, et al. Minimal intensity physical activity (standing and walking) of longer duration improves insulin action and plasma lipids more than shorter periods of moderate to vigorous exercise (cycling) in sedentary subjects when energy expenditure is comparable. PLoS One 2013;8:e55542.

31 Zeigler ZS, Swan PD, Bhammar DM, et al. Walking Workstation Use Reduces Ambulatory Blood Pressure in Adults with Prehypertension. Journal of Physical Activity and Health 2015; 12:S119-27.

32 Duvivier BM, Schaper NC, Hesselink MK, et al. Breaking sitting with light activities vs structured exercise: a randomised crossover study demonstrating benefits for glycaemic control and insulin sensitivity in type 2 diabetes. Diabetologia 2017;60:490-8.

33 Duvivier B, Schaper NC, Koster A, et al. Benefits of substituting sitting with standing and walking in free-living conditions for cardiometabolic risk markers, cognition and mood in overweight adults. Front Physiol 2017;8.

34 Thorp AA, Kingwell BA, Sethi P, et al. Alternating bouts of sitting and standing attenuate postprandial glucose responses. Med Sci Sports Exerc 2014;46:2053-61.

35 Bailey DP, Locke CD. Breaking up prolonged sitting with light-intensity walking improves postprandial glycemia, but breaking up sitting with standing does not. J Sci Med Sport 2015;18.

36 Zeigler ZS, Swan PD, Bhammar DM, et al. Walking workstation use reduces ambulatory blood pressure in adults with prehypertension. J Phys Act Health 2015;12:S119-27

37 Dempsey PC, Blankenship JM, Larsen RN, et al. Interrupting prolonged sitting in type 2 diabetes: nocturnal persistence of improved glycaemic control. Diabetologia 2017;60:499-507

38 Bailey DP, Broom DR, Chrismas BC, et al. Breaking up prolonged sitting time with walking does not affect appetite or gut hormone concentrations but does induce an energy deficit and suppresses postprandial glycaemia in sedentary adults. App/ Physiol Nutr Metab 2016;41:324-31.

39 Nishida $Y$, Higaki Y, Tokuyama $K$, et al. Effect of mild exercise training on glucose effectiveness in healthy men. Diabetes Care 2001;24:1008-13.
40 Nishida Y, Matsubara T, Tobina T, et al. Effect of low-intensity aerobic exercise on insulin-like growth factor-I and insulin-like growth factor-binding proteins in healthy men. Int J Endocrinol 2010;1-8.

41 Okano G, Sato Y, Murata Y. effect of mild walk habit on body composition, blood pressure and serum lipids. Japanese Journal of Physical Fitness and Sports Medicine 1990;39:315-23

42 Paoli A, Pacelli QF, Moro T, et al. Effects of high-intensity circuit training, low-intensity circuit training and endurance training on blood pressure and lipoproteins in middleaged overweight men. Lipids Health Dis 2013;12:131.

43 Skoro-Kondza L, Tai SS, Gadelrab R, et al. Community based yoga classes for type 2 diabetes: an exploratory randomised controlled trial. BMC Health Serv Res 2009;9:33.

44 Krause M, Rodrigues-Krause J, O'Hagan C, et al. The effects of aerobic exercise training at two different intensities in obesity and type 2 diabetes: implications for oxidative stress, low-grade inflammation and nitric oxide production. Eur J Appl Physiol 2014;114:251-60.

45 de Lannoy L, Clarke J, Stotz PJ, et al. Effects of intensity and amount of exercise on measures of insulin and glucose: Analysis of inter-individual variability. PLoS One 2017;12:e0177095

46 Evenson KR, Wen F, Herring AH. Associations of accelerometry-assessed and selfreported physical activity and sedentary behavior with all-cause and cardiovascular mortality among US adults. Am J Epidemio/ 2016;184:621-32.

47 Loprinzi PD, Lee H, Cardinal BJ. Evidence to support including lifestyle light-intensity recommendations in physical activity guidelines for older adults. Am J Health Promot 2015:29:277-84

48 Fishman El, Steeves JA, Zipunnikov V, et al. Association between objectively measured physical activity and mortality in NHANES. Med Sci Sports Exerc 2016;48:1303-11.

49 McAteer A, Hravnak M, Chang Y, et al. The relationships between BNP and neurocardiac injury severity, noninvasive cardiac output, and outcomes after aneurysmal subarachnoid hemorrhage. Biol Res Nurs 2017;19:531-7.

50 Matthews CE, Keadle SK, Troiano RP, et al. Accelerometer-measured dose-response for physical activity, sedentary time, and mortality in US adults. Am J Clin Nutr 2016;104:1424-32.

51 Laaksonen DE, Lakka HM, Salonen JT, et al. Low levels of leisure-time physical activity and cardiorespiratory fitness predict development of the metabolic syndrome. Diabetes Care 2002;25:1612-8.

52 Mekary RA, Willett WC, Hu FB, et al. Isotemporal substitution paradigm for physical activity epidemiology and weight change. Am J Epidemiol 2009;170:519-27.

53 Hu G, Eriksson J, Barengo NC, et al. Occupational, commuting, and leisure-time physical activity in relation to total and cardiovascular mortality among finnish subjects with type 2 diabetes. Circulation 2004;110:666-73.

54 Ekelund U, Brage S, Griffin SJ, et al. Objectively measured moderate- and vigorousintensity physical activity but not sedentary time predicts insulin resistance in high-risk individuals. Diabetes Care 2009:32:1081-6.

55 Hamer M, de Oliveira C, Demakakos P. Non-exercise physical activity and survival: english longitudinal study of ageing. Am J Prev Med 2014;47:452-60.

56 Sabia S, Dugravot A, Kivimaki M, et al. Effect of intensity and type of physical activity on mortality: results from the Whitehall II cohort study. Am J Public Health 2012;102:698-704.

57 Pate RR, et al. Physical Activity and Public Health. JAMA 1995;273:402

58 Bey L, Hamilton MT. Suppression of skeletal muscle lipoprotein lipase activity during physical inactivity: a molecular reason to maintain daily low-intensity activity. J Physiol 2003;551:673-82

592018 Physical Activity Guidelines Advisory Committee. 2018 Physical Activity Guidelines Advisory Committee Scientific Report. Washington, DC: U.S. Department of Health and Human Services. 2018.

60 Chastin SF, Palarea-Albaladejo J, Dontje ML, et al. Combined effects of time spent in physical activity, sedentary behaviors and sleep on obesity and cardiometabolic health markers: a novel compositional data analysis approach. PLoS One 2015; 10:e0139984. 